\title{
GW23-e2105 HRAMP1 MODIFIED MSCS IMPROVE CARDIAC FUNCTION AND INHIBIT NEOINTIMAL PROLIFERATION IN THE CAROTID ANGIOPLASTY AND MYOCARDIAL INFARCTION RABBIT MODEL
}

doi:10.1136/heartjnl-2012-302920a.10

Bei Shi, Xianping Long, Ranzun Zhao, Zhijing Liu, Dongmei Wang, Guanxue Xu, Bei Shi. the First Affiliated Hospital of Zunyi Medical College

Objectives Although transplanting mesenchymal stem cells (MSCs) can improve cardiac function and contribute to endothelial recovery in a damaged artery, natural MSCs may induce neointimal hyperplasia by directly or indirectly acting on vascular smooth muscle cells (VSMCs). Here, we investigated the effects of MSCs overexpressing the human receptor activity-modifying protein 1 (hRAMP1) on heart function and artery repair in rabbit models of myocardial infarction reperfusion and carotid artery injury.

Methods MSCs transfected with a recombinant adenovirus containing the hRAMP1 gene (EGFP-hRAMP1-MSCs) were injected into the rabbit models via the ear vein at $24 \mathrm{~h}$ after carotid artery injury and myocardial infarction.

Results Seven days post EGFP-hRAMP1-MSC transplantation, the cells that expressed both EGFP and CD31 were detected in the neointima of the damaged artery via immunofluorescence. EGFPhRAMP1 expression was observed in the injured artery and infarcted myocardium by western blot analysis, confirming that the engineered MSCs were targeted the injured artery and infarcted myocardium and expressed hRAMP1 protein. Compared with the EGFP-MSCs group, the EGFP-hRAMP1-MSCs group had a significantly smaller infarcted area and improved cardiac function by 28 day after cell transplantation, as detected by triphenyltetrazolium chloride (TTC) staining and echocardiography. Additionally, arterial HE staining revealed that the area of the neointima and the area ratio of intima-media were significantly decreased in the EGFPhRAMP1-MSCs group.

Conclusions Therefore, compared with natural MSCs, EGFPhRAMP1-engineered MSCs improved infarcted heart function and endothelial recovery from artery injury more efficiently, which will provide valuable information for the development of MSCs-based therapy. 